\title{
Contribution of microtopography and hydroperiod to the natural regeneration of Avicennia germinans in a restored mangrove forest
}

\section{Contribución del hidroperiodo y la microtopografía a la regeneración natural de Avicennia germinans en un sitio de restauración ecológica}

\author{
Rosela Pérez-Ceballos ${ }^{1 *}$, Stephanie Echeverría-Ávila², Arturo Zaldívar-Jiménez ${ }^{2,3}$, \\ Tomás Zaldívar-Jiménez ${ }^{2}$, Jorge Herrera-Silveira ${ }^{3}$ \\ ${ }^{1}$ Instituto de Ciencias del Mar y Limnología, estación El Carmen, Universidad Nacional Autónoma de México, \\ Carretera Carmen Puerto Real, km 9.5, CP 24157, Ciudad del Carmen, Campeche, México. \\ 2 ATEC Asesoría Técnica y Estudios Costeros SCP, Calle 63b, no. 221, fraccionamiento Yucalpetén, CP 97238 , \\ Mérida, Yucatán, México. \\ ${ }^{3}$ Centro de Investigación y Estudios Avanzados del Instituto Politécnico Nacional (CINVESTAV), \\ Unidad Mérida, km 6 Antigua carretera a Progreso, Apdo. Postal 73, Cordemex, CP 97310, Mérida, \\ Yucatán, México. \\ * Corresponding author. E-mail: rosela.perezc@gmail.com
}

\begin{abstract}
We evaluated the success of the ecological restoration of a degraded mangrove forest by monitoring the natural regeneration of Avicennia germinans (black mangrove). The restoration actions focused on hydrological rehabilitation by desilting tidal channels and modifying the microtopography to enhance or restore water flow. As a result of these efforts, a population of black mangrove established naturally. Six sampling sites including restored and reference areas were established. We measured seedling survival; tree density, height, and growth rate; and the physical and chemical characteristics of soil and water. According to a redundancy analysis, microtopography and hydroperiod contributed the most to the establishment and development of the black mangrove. Microtopography ranged from 0.08 to $0.14 \mathrm{~m}$ above sea level. In the case of the hydroperiod, water level ranged from 0.11 to $0.14 \mathrm{~m}$, flooding duration ranged from 439 to $529 \mathrm{~h}$ per month, and frequency was 7 to 8 floods per month. Our results highlight the contribution of microtopography and hydroperiod to the natural establishment of black mangrove. It is important to consider these 2 variables in mangrove ecological restoration.
\end{abstract}

Key words: black mangrove, hydrologic rehabilitation, environmental monitoring, recruitment, karst setting.

RESUMEN. Se evaluó la restauración ecológica de un manglar degradado por actividades antrópicas mediante el seguimiento de la regeneración natural de Avicennia germinans (mangle negro). La principal acción de restauración fue la rehabilitación hidrológica, la cual consistió en el desazolve de canales de marea y la modificación de la topografía para reactivar el flujo hidrológico. Como resultado de estas acciones, una población de A. germinans se estableció de manera natural. Se establecieron 6 sitios de muestreo que incluyeron áreas restauradas y de referencia (manglar natural). Se midieron la superviviencia de las plántulas; la densidad, altura y tasa de crecimiento de las plántulas; y las caractrerísticas físicas y químicas del suelo y del agua. El análisis de redundancia demostró que la microtopografía y el hidroperiodo fueron las variables de mayor contribución al establecimiento y desarrollo de A. germinans. El intervalo de microtopografía fue de 0.08 a $0.14 \mathrm{~m}$ sobre el nivel del mar. En el caso del hidroperiodo, el intervalo para el nivel de inundación fue de 0.11 a $0.14 \mathrm{~m}$, para el tiempo de inundación fue de 439 a 529 h por mes y para la frecuencia de inundación fue de 7 a 8 inundaciones por mes. Los resultados indicaron la contribución de la microtopografía y el hidroperiodo al establecimiento natural del mangle negro. Es importante considerar estas 2 variables en la restauración ecológica de manglares.

Palabras clave: mangle negro, rehabilitación hidrológica, monitoreo ambiental, reclutamiento, escenario cárstico.

\section{INTRODUCTION}

Mangrove ecosystems are defined by their hydrology and type of vegetation, which is adapted to varying inundation, salinity, and reduced soil conditions (Kuenzer et al. 2011). The different types of mangroves provide refuge, nursery, and foraging areas for fishes (Vaslet et al. 2015), and play a role in nutrient input and removal processes (MoroyoquiRojo et al. 2015).

\section{INTRODUCCIÓN}

Los manglares son un ecosistema definido por su hidrología y tipo de vegetación, la cual está adaptada a variaciones de inundación, salinidad y condiciones reducidas del suelo (Kuenzer et al. 2011). Los diferentes tipos de manglar actúan como zonas de refugio, crianza y alimentación para los peces (Vaslet et al. 2015), además de que juegan un papel en los procesos de importación y exportación de nutrientes (Moroyoqui-Rojo et al. 2015). 
Resources, regulators, and hydroperiod are environmental factors that determine the structure and function of mangroves (Twilley and Rivera-Monroy 2005). Resources (nutrients) are associated with water sources and microbial activities (Cardona-Olarte et al. 2006, Whigham et al. 2009, Reef et al. 2010). Regulators (stress factors), such as salinity, sulfides, and temperature, have an affect on the ecophysiology of mangrove plants. Salinity is one of the main factors regulating recruitment (successful incorporation into a population of new individuals) (McKee 1995, Ricklefs and Miller 2000) and mangrove development (Feller et al. 2010). Hydroperiod is defined by inundation frequency (number of floods in a given period of time), flooding duration (how long flooding persists), and water level, and is considered a key factor for mangrove growth (Krauss et al. 2008, Bashan et al. 2013).

Topography and hydroperiod maintain a direct relationship with soil properties and mangrove vegetation (Komiyama et al. 1996, Flores-Verdugo et al. 2006, Dahdouh-Guebas et al. 2007). Topography is therefore another important factor that defines the hydroperiod and allows the establishment of different mangrove species (Flores-Verdugo et al. 2007). The subtropical mangrove species from the American continent differ in their tolerance to flooding. Differences in growth and survival are attributed to diverse physiological factors since each mangrove species responds to local environments and ecological controls (Monroy-Torres et al. 2014).

Regeneration occurs via biological processes and under favorable environmental conditions. A disturbance can limit mangrove growth and development. Hence, the regeneration of mangroves is directly influenced by the reigning environmental conditions that will affect the stages of plant development (Sousa et al. 2003). Changes in hydroperiod can thus affect growth and survival during the seedling and juvenile stages (McKee 1995, Kitaya et al. 2002, He et al. 2007). In a perturbed habitat, seedling recruitment occurs when the values of the regulators are close to those of a natural ecosystem (Bosire et al. 2008). Consequently, this indicator can help us understand the response of a mangrove forest to a natural disturbance and can be used as a measure of ecological restoration.

Natural and human-caused disturbances have different effects (positive, negative, or neutral) on mangroves (FloresVerdugo et al. 2006). The time a mangrove ecosystem takes to recover will depend on its resilience or its historical development trajectory (SER 2004). When it can no longer recover independently, some active intervention is necessary. Restoration activities should be designed taking into consideration information of the pre-existing structure, composition, and function of the ecosystem or of a reference ecosystem (SER 2004). Mangrove restoration activities include hydrologic rehabilitation, reforestation, or a combination of both (SER 2004). In any case, each method requires basic knowledge of
Los recursos, los reguladores y el hidroperiodo son factores ambientales que determinan la estructura y función de los manglares (Twilley y Rivera-Monroy 2005). Los recursos (nutrientes) están asociados con las fuentes de agua y la actividad microbiana (Cardona-Olarte et al. 2006, Whigham et al. 2009, Reef et al. 2010). Los reguladores (factores de estrés) como la salinidad, el sulfuro y la temperatura tienen un efecto sobre la ecofisiología de las plantas de manglar. La salinidad es uno de los reguladores más importantes para el reclutamiento (establecimiento exitoso de la incorporación de nuevos individuos a la población) (McKee 1995, Ricklefs y Miller 2000) y el desarrollo del manglar (Feller et al. 2010). El hidroperiodo está definido por la frecuencia de inundación (número de inundaciones por tiempo), el periodo de inundación (tiempo en el que permanece inundado) y el nivel de inundación, y es considerado el factor clave en el crecimiento de los manglares (Krauss et al. 2008, Bashan et al. 2013).

La topografía y el hidroperiodo mantienen una relación directa con las propiedades del suelo y con la vegetación de manglar (Komiyama et al. 1996, Flores-Verdugo et al. 2006, Dahdouh-Guebas et al. 2007). Por lo tanto, la topografía es otro factor importante que define el hidroperiodo y permite el establecimiento diferenciado de las especies de mangle (Flores-Verdugo et al. 2007). Las diferentes especies subtropicales de mangle del continente americano difieren ampliamente en su tolerancia a la inundación. Las diferencias en el crecimiento y la supervivencia de las especies son atribuidas a los distintos factores fisiológicos, ya que cada especie de mangle responde a los ambientes locales y los controles ecológicos (Monroy-Torres et al. 2014).

La regeneración ocurre mediante procesos biológicos y en condiciones ambientales favorables; sin embargo, después de una perturbación, la regeneración puede verse afectada al limitarse el crecimiento y desarrollo de la comunidad de manglar. Las condiciones ambientales que permanezcan en el hábitat perturbado influenciarán de forma directa la regeneración del manglar, ya que influenciarán el desarrollo de las etapas de crecimiento del mangle (Sousa et al. 2003). Es por ello que los cambios en el hidroperiodo pueden afectar el crecimiento y la supervivencia durante la etapa de plántula y la etapa juvenil (McKee 1995, Kitaya et al. 2002, He et al. 2007). En un hábitat perturbado, el reclutamiento de plántulas se produce cuando los reguladores presentan valores cercanos a los de un ecosistema natural (Bosire et al. 2008). El reclutamiento de las plántulas del manglar será un indicador que ayudará a entender la respuesta del manglar a una perturbación natural y servirá como una medida de la restauración ecológica.

Los disturbios antrópicos y no antrópicos tienen diversos efectos (positivos, negativos o neutrales) sobre los manglares (Flores-Verdugo et al. 2006). El tiempo de recuperación de un ecosistema de manglar dependerá de su capacidad para restablecerse por sí solo al estado anterior a la alteración o a su trayectoria histórica de desarrollo (SER 2004). Cuando un ecosistema de manglar ya no se puede restablecer por sí solo, 
the regional hydroperiod, which in turn requires knowledge of the tidal regime and microtopography (Flores-Verdugo et al. 2007). Restoration success will depend on the measures implemented, but environmental monitoring is necessary to determine the recovery trajectory (Zaldívar-Jiménez et al. 2010).

In the Yucatán Peninsula (Mexico), anthropogenic activities have reduced the ability of mangroves to respond to disturbances such as storms and hurricanes (Zaldívar-Jiménez et al. 2010, Adame et al. 2013). The hydrologic system has been modified by roads that disrupt water flow, by changes to barrier islands, and by the dumping of dredged sediments (Teutli-Hernández 2008, García de Fuentes et al. 2011). In the state of Yucatán, several restoration projects have been carried out along the coast but have not been as successful as expected because the hydrologic conditions and sedimentary characteristics necessary for mangrove regeneration were not taken into account (Zaldívar-Jiménez et al. 2010). To implement mangrove restoration strategies based on hydrologic rehabilitation and monitor the natural regeneration, a restoration program was developed that focused on the desilting of tidal channels to rehabilitate the hydroperiod and on modifying the topography to improve natural recruitment and development of the black mangrove (Avicennia germinans) (Zaldívar-Jiménez et al. 2010). The purpose of the present study was to assess the natural regeneration of A. germinans by monitoring its growth and development in relation to hydroperiod, salinity, topography, and soil properties after the restoration actions. Our hypothesis is that the natural regeneration of a mangrove species differs depending on environmental gradients.

\section{MATERIALS AND METHODS}

\section{Study area}

The study area is located within the Chelem Lagoon system, in the northern part of the Yucatán Peninsula (Fig. 1). The soils are of karstic origin and the area is characterized by high evaporation and scant rainfall (mean annual rainfall of $587 \mathrm{~mm}$ ). Mean annual temperature is $25.8^{\circ} \mathrm{C}$; during the coldest season the temperature is $22.5^{\circ} \mathrm{C}$. The climate is mainly hot and dry, but hot and humid in summer (Orellana and Espadas 2003).

The mangrove habitat in the study area has been affected by anthropogenic activities. Dredged sediments from the port of Yucalpetén were dumped in the area and modified the topography, hydroperiod, and consequently the physical and chemical properties of the soil, affecting the structure of the mangrove forest community. Prior to the ecological restoration, bulk density was $0.81 \pm 0.19 \mathrm{~g} \mathrm{~cm}^{-3}$ and litterfall was $2.94 \pm 0.25 \mathrm{~g} \mathrm{~m}^{-2} \mathrm{~d}^{-1}$ (Adame et al. 2013).

Mangrove degradation was primarily caused by the silting up of natural channels because of the disposal of es necesario contribuir con acciones de restauración. El diseño de las acciones de restauración se establece con base en los conocimientos sobre la estructura, composición y funcionamiento preexistentes del ecosistema afectado o de un ecosistema de referencia (SER 2004). Las acciones de restauración de los manglares pueden incluir la rehabilitación hidrológica, la reforestación o la combinación de ambas (SER 2004). En cualquier caso, cada método requiere de un conocimiento básico del hidroperiodo de la región, que a su vez requiere del conocimiento del régimen de mareas y la microtopografía (Flores-Verdugo et al. 2007). El éxito de la restauración dependerá de las actividades que se efectúen, pero es necesario el monitoreo ambiental para determinar la trayectoria de restauración que sigue el manglar (ZaldívarJiménez et al. 2010).

En la península de Yucatán (México), las actividades antrópicas han reducido la capacidad de respuesta de los manglares a perturbaciones como tormentas y huracanes (Zaldívar-Jiménez et al. 2010, Adame et al. 2013). Las modificaciones al sistema hidrológico se deben al cruce de carreteras que cortan los flujos de agua, a los rompimientos de las islas de barrera y a las obras de relleno (Teutli-Hernández 2008, García de Fuentes et al. 2011). En el estado de Yucatán, se han realizado numerosos proyectos de restauración a lo largo de la costa, aunque no con el éxito esperado debido a que no se tomaron en cuenta las condiciones hidrológicas y las características del suelo necesarias para la regeneración del manglar (Zaldívar-Jiménez et al. 2010). Con la intención de implementar la estrategia de restauración de manglares mediante la rehabilitación hidrológica y monitorear la regeneración natural, se desarrolló un programa de acciones de restauración que incluyeron el desazolve de canales de marea para rehabilitar el hidroperiodo y la modificación de la topografía para mejorar el reclutamiento y desarrollo natural del mangle negro (Avicennia germinans) (Zaldívar-Jiménez et al. 2010). Considerando lo anterior, el propósito de este estudio fue evaluar la regeneración natural de A. germinans mediante el monitoreo de su crecimiento y desarrollo en respuesta al hidroperiodo, la salinidad, la topografía y las características del suelo después de las acciones de restauración. La hipótesis de este estudio plantea que la regeneración natural de una especie de mangle tiene una respuesta diferencial a los gradientes ambientales.

\section{MATERIALES Y MÉTODOS}

\section{Área de estudio}

El área de estudio se encuentra en la parte norte de la península de Yucatán, dentro del sistema lagunar de Chelem (Fig. 1). Los suelos son de origen cárstico y la zona se caracteriza por la alta evaporación y baja precipitación (media anual de $587 \mathrm{~mm}$ ). La temperatura media anual es de $25.8^{\circ} \mathrm{C}$; en la temporada más fría la temperatura es de $22.5^{\circ} \mathrm{C}$. El clima del lugar es principalmente muy cálido y seco, y en verano es cálido y lluvioso (Orellana y Espadas 2003). 


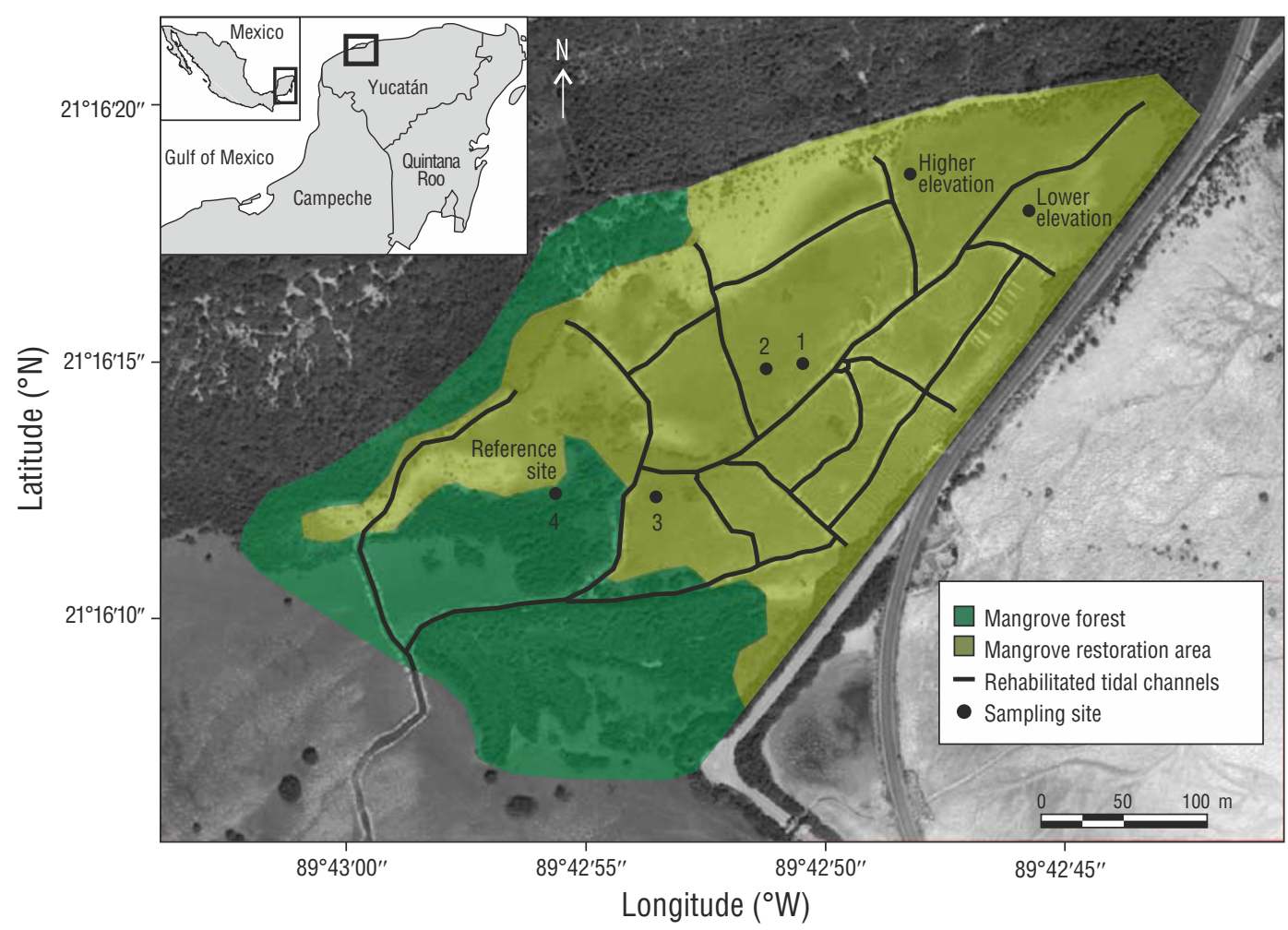

Figure 1. Map of the study area showing the location of the 6 sampling sites: 3 restored mangrove sites (sites 1-3), 1 natural mangrove site (reference, site 4), and 2 sites with higher and lower elevation relative to the other sites.

Figura 1. Mapa del área de estudio donde se muestra la ubicación de los 6 sitios de muestreo: 3 sitios de manglar en el área de restauración (sitios 1-3), 1 sitio de manglar natural (referencia, sitio 4) y 2 sitios de mayor y menor elevación con respecto a los otros sitios.

degraded material in surrounding areas. Hydrologic rehabilitation thus became the main restoration action. Rehabilitation activities were carried out between 2009 and 2010, and consisted in manually desilting tidal channels and modifying the topography. With the help of the Chelem community, $2,000 \mathrm{~m}$ of tidal channels were desilted to restore the hydrology in the degraded area. As a result of this effort, A. germinans propagules and recruitment were subsequently observed in the restoration area.

\section{Sampling sites}

To determine the characteristics of the soil, hydrology, and natural regeneration of $A$. germinans, permanent sampling units were established at 6 sites (Fig. 1). Sites 1, 2, and 3 , located within the area selected for restoration activities, had dead trees and were void of vegetation at the beginning of the study. At site 4, the adjacent reference site, the mangrove forest was in a natural state. Two other reference sites were established, one at a higher elevation $(+0.24$ meters above sea level [masl]) and the other at a lower elevation $(-0.04$ masl) relative to the other sites. The latter was permanently flooded and seedlings were not observed at either of these 2 sites.
El área de estudio es una zona de manglar impactada por actividades antrópicas. En este sitio fueron arrojados sedimentos de dragado del puerto de Yucalpetén (marina), lo que modificó la topografía, el hidroperiodo y, por consiguiente, los factores fisicoquímicos del suelo que influyen sobre la estructura de la comunidad de manglar. Estas modificaciones dieron como resultado la degradación y muerte de los manglares. Antes de la restauración ecológica, la densidad aparente del suelo era de $0.81 \pm 0.19 \mathrm{~g} \mathrm{~cm}^{-3}$ y la acumulación de hojarasca era de $2.94 \pm 0.25 \mathrm{~g} \mathrm{~m}^{-2} \mathrm{~d}^{-1}$ (Adame et al. 2013).

La causa principal de la degradación de los manglares en el área de estudio se debió al azolvamiento de los canales naturales por la disposición de material degradado en zonas circundantes. Por ello, la rehabilitación hidrológica fue la principal actividad de restauración ecológica. Las acciones de rehabilitación hidrológica se realizaron entre 2009 y 2010, y consistieron en el desazolve manual de canales de marea y la modificación de la topografía. Con el involucramiento de la comunidad de Chelem, se desazolvaron, en total, 2,000 m de canales de marea para restablecer el flujo del agua lagunar dentro del área degradada. Como resultado de las acciones de rehabilitación hidrológica, se registró la entrada de propágulos y el reclutamiento de A. germinans en el área de restauración. 


\section{Soil characteristics}

The characteristics of the water and soil were monitored from 2008 to 2012. For the analysis, data were divided into those obtained before (2008 and 2009) and after (2010 to 2012) implementing the restoration activities. Soil was characterized at each site based on bulk density, organic matter content, and microtopography (Chen and Twilley 1999). Bulk density and organic matter were analyzed in 2008 (before) and 2013 (after). The microtopography was classified in 2013 using a STONEX STS2-R Total Station; elevation was established relative to mean sea level using datum WGS84.

\section{Hydrologic characteristics}

Porewater salinity and hydroperiod were measured at each site. Soil salinity was measured in situ every 2 months with a YSI model 30 multiparameter probe. Hydroperiod was determined with a pressure sensor (HOBO, Onset Computer Corporation, USA) installed in the study area; the collected data were converted into water levels. Measurements were carried out continuously over one year and used to determine inundation frequency, water level, and flooding duration.

\section{Natural regeneration of Avicennia germinans}

To assess the natural regeneration of A. germinans, permanent sampling units of $314.16 \mathrm{~m}^{2}$ were established. All individuals within each unit were labelled with a plaque for subsequent monitoring. Plant species, identification number, and height were recorded. Height $(\mathrm{cm})$ was measured with a graduated ruler from the soil to the highest apex of the plant. Measurements were carried out every 6 months for plant density, relative growth rate in height (RGRH), and survival. Density was calculated as the number of individuals per unit (i.e., per $0.01 \mathrm{ha})$. RGRH ( $\mathrm{mm} \mathrm{d}^{-1}$ ) was measured relative to time one (Rodríguez-Ramírez et al. 2004).

\section{Statistical analysis}

Two-way ANOVA was used to determine the differences among sites and restoration period (before or after) with respect to water and soil variables. A redundancy analysis (RDA) was carried out to determine the association between the response (natural regeneration) variables and the soil and hydrologic variables. RDA explains the response variables as a function of the explanatory variables. It can also be seen as an extension of the principal components analysis. What distinguishes RDA is that it produces a predictive model based on response variables with a linear combination of the predictor variables. The total proportion of variance can be explained by the linear combination of the predictor variables (Tabachnick and Fidell 1996, Legendre and Legendre 1998). Analyses were done using the R (v2.11.1) command console and Tinn-R editor (R Core Team 2012).

\section{Sitios de muestreo}

Para determinar las características del suelo, la hidrología y la regeneración natural de $A$. germinans, se establecieron unidades de muestreo permanentes en 6 sitios (Fig. 1). Los sitios 1, 2 y 3 se ubicaron dentro del área con acciones de restauración, y eran zonas degradadas con árboles muertos y sin vegetación al inicio del estudio. El sitio 4 fue una zona aledaña y se consideró como referencia porque la vegetación del manglar estaba en estado natural. Se establecieron 2 sitios más de referencia, uno de mayor elevación $(+0.24$ metros sobre el nivel del mar [msnm]) y otro, permanentemente inundado, de menor elevación $(-0.04 \mathrm{msnm})$ con respecto a los sitios de manlgar; ninguno de estos 2 sitios tenía plántulas.

\section{Características del suelo}

El monitoreo de las características del agua y suelo se realizó de 2008 a 2012. Para el análisis, los datos se dividieron de acuerdo con el momento de las acciones de restauración: antes (2008 y 2009) y después (de 2010 a 2012). La caracterización del suelo se determinó para cada sitio mediante la densidad aparente del suelo, la cantidad de materia orgánica y la microtopografía (Chen y Twilley 1999). El análisis de la densidad aparente y la materia orgánica se realizaron en 2008 (antes) y en 2013 (después). La microtopografía se realizó en 2013 con una estación total STONEX STS2-R, y las alturas del suelo fueron establecidas con respecto al nivel medio del mar utilizando el datum WGS84.

\section{Características hidrológicas}

En cada sitio, se midieron la salinidad intersticial y el hidroperiodo. La salinidad del suelo se midió in situ cada 2 meses con una sonda multiparamétrica YSI modelo 30. El hidroperiodo se determinó con un medidor de presión (HOBO, Onset Computer Corporation, EUA) que se instaló en el área de estudio, y los datos recolectados se convirtieron a niveles de inundación. Las mediciones se realizaron continuamente durante un año y se utilizaron para determinar la frecuencia, el nivel y el periodo de inundación.

\section{Regeneración natural de Avicennia germinans}

Para evaluar la regeneración natural de A. germinans, se establecieron unidades de muestreo permanentes de $314.16 \mathrm{~m}^{2}$. Todos los individuos presentes en cada unidad fueron etiquetados con una placa de identificación para su seguimiento. Los datos registrados para cada plántula fueron la especie, el número de identificador y la altura. La altura $(\mathrm{cm})$ se midió con una regla graduada, desde la superficie del suelo hasta el ápice más alto de la plántula. Las mediciones se realizaron cada 6 meses para determinar la densidad, tasa relativa de crecimiento en altura (TRCA) y supervivencia. La densidad se calculó como el número de 


\section{RESULTS}

\section{Soil characteristics}

Changes in bulk density $(P<0.01)$ were observed at sites 1,2 , and 3 (restoration area) during the study period, decreasing from $1.33 \pm 0.23 \mathrm{~g} \mathrm{~cm}^{-3}$ (before implementing restoration actions) to $0.60 \pm 0.01 \mathrm{~g} \mathrm{~cm}^{-3}$ (after). It also decreased at site 4 (reference), from $0.95 \pm 0.18$ to $0.55 \pm 0.08 \mathrm{~g} \mathrm{~cm}^{-3}$ (Table 1). Changes in bulk density were not recorded at the higher and lower elevation sites. Organic matter content differed among sites $(P<0.0001)$, the lower and higher elevation sites having the highest and lowest values $(23 \pm 0.40 \%$ and $14 \pm 0.39 \%$, respectively) (Table 1 ).

Topographic elevation at site 4 was $+0.14 \pm 0.02$ masl, and at the higher and lower elevation sites it was $+0.24 \pm 0.03$ and $-0.04 \pm 0.01$ masl, respectively. The natural regeneration of A. germinans occurred between 0.087 and $0.14 \mathrm{~m}$ above mean sea level (Table 2). Seedling establishment was not observed at the higher and lower elevation sites.

\section{Hydrologic characteristics}

Differences in salinity were observed between sites $(P<0.0001)$ and between restoration time $(P<0.003)$. After the restoration actions, the lowest salinity value $(64.50 \pm$ 1.80) was recorded at site 4 (reference) and the highest $(75.90 \pm 2.61)$ at the higher elevation site (Table 1).

Differences in water level and flooding duration were observed between sites $(P<0.05)$ and between restoration time $(P<0.05)$ (Table 2). Four floods per months were recorded at the lower elevation site, whereas 7 to 8 floods per months were recorded at the other sites. Water level was highest $(0.28 \mathrm{~m})$ at the lower elevation site and lowest $(0.08 \mathrm{~m})$ at the higher elevation site. Mean water level was $0.14 \mathrm{~m}$ at sites $1-3$ and $0.11 \mathrm{~m}$ at site 4 (reference). Mean flooding duration decreased as elevation increased. It was plántulas por unidad (0.01 ha). El crecimiento se midió a partir de la TRCA $\left(\mathrm{mm} \mathrm{d}^{-1}\right)$, la cual se calculó a partir del tiempo uno de cada sitio (Rodríguez-Ramírez et al. 2004).

\section{Análisis estadístico}

Los datos se evaluaron con el análisis de varianza de 2 vías para determinar las diferencias entre sitios y tiempo (antes o después) de la restauración con respecto a las variables del agua y suelo. Para determinar la asociación de las variables de respuesta (regeneración natural del manglar) con las variables del suelo y las variables hidrológicas, se llevó a cabo un análisis de redundancia (RDA, por sus siglas en inglés). El RDA explica las variables de respuesta como una función de las variables explicativas. Este análisis también puede verse como una extensión del análisis de componentes principales. Lo que distingue al RDA es que desarrolla un modelo predictivo a partir de las variables de respuesta mediante una combinación lineal de las variables predictivas. La proporción total de la varianza puede ser explicada en respuesta a una combinación lineal de las variables predictoras (Tabachnick y Fidell 1996, Legendre y Legendre 1998). Se utilizaron la consola de comandos del programa $\mathrm{R}$ (v2.11.1) y el editor de comandos Tinn-R (R Core Team 2012).

\section{RESULTADOS}

\section{Características del suelo}

La densidad aparente del suelo cambió con las acciones de restauración $(P<0.01)$. Antes de las acciones de restauración, el promedio de la densidad aparente en los sitios 1, 2 y 3 fue de $1.33 \pm 0.23 \mathrm{~g} \mathrm{~cm}^{-3}$ y después de las acciones fue de $0.60 \pm 0.01 \mathrm{~g} \mathrm{~cm}^{-3}$. En el sitio 4 (referencia) también se observó una disminución, de $0.95 \pm 0.18 \mathrm{~g} \mathrm{~cm}^{-3}$ a $0.55 \pm$ $0.08 \mathrm{~g} \mathrm{~cm}^{-3}$ (Tabla 1). Los sitios de mayor y menor elevación

Table 1. Comparison of the soil variables before and after implementing restoration actions (mean $\pm \mathrm{SE}$ ).

Tabla 1. Comparación de las variables del suelo antes y después de las acciones de restauración (media \pm error estándar).

\begin{tabular}{|c|c|c|c|c|c|c|c|}
\hline \multirow[b]{2}{*}{ Restoration variable } & \multirow[b]{2}{*}{$n$} & \multicolumn{6}{|c|}{ Site } \\
\hline & & 1 & 2 & 3 & 4 & $\begin{array}{l}\text { Lower } \\
\text { elevation }\end{array}$ & $\begin{array}{l}\text { Higher } \\
\text { elevation }\end{array}$ \\
\hline \multicolumn{8}{|l|}{ Before } \\
\hline Salinity & 32 & $78.40 \pm 3.60$ & $79.50 \pm 3.80$ & $70.00 \pm 2.40$ & $71.60 \pm 1.80$ & $67.40 \pm 4.00$ & $85.80 \pm 4.30$ \\
\hline Bulk density $\left(\mathrm{g} \mathrm{cm}^{-3}\right)$ & 8 & $1.15 \pm 0.60$ & $1.05 \pm 0.53$ & $1.80 \pm 0.71$ & $0.95 \pm 0.18$ & $0.47 \pm 0.02$ & $1.89 \pm 0.05$ \\
\hline Organic matter $(\%)$ & 8 & $16.90 \pm 0.93$ & $19.10 \pm 1.29$ & $16.90 \pm 1.37$ & $17.40 \pm 0.25$ & $22.50 \pm 0.53$ & $13.50 \pm 0.55$ \\
\hline \multicolumn{8}{|l|}{ After } \\
\hline Salinity & 40 & $71.70 \pm 3.50$ & $72.70 \pm 3.30$ & $66.50 \pm 1.70$ & $64.50 \pm 1.80$ & $63.40 \pm 2.30$ & $75.90 \pm 2.60$ \\
\hline Bulk density $\left(\mathrm{g} \mathrm{cm}^{-3}\right)$ & 12 & $0.63 \pm 0.09$ & $0.59 \pm 0.05$ & $0.60 \pm 0.14$ & $0.55 \pm 0.09$ & $0.40 \pm 0.01$ & $1.81 \pm 0.03$ \\
\hline Organic matter $(\%)$ & 12 & $17.30 \pm 1.67$ & $19.30 \pm 0.73$ & $20.00 \pm 2.00$ & $17.80 \pm 1.18$ & $23.50 \pm 0.57$ & $14.50 \pm 0.50$ \\
\hline
\end{tabular}


highest at the lower elevation site $\left(680 \mathrm{~h} \mathrm{mo}^{-1}\right)$, followed by sites $1-3\left(529 \mathrm{~h} \mathrm{mo}^{-1}\right)$, site $4\left(439 \mathrm{~h} \mathrm{mo}^{-1}\right)$, and the higher elevation site $\left(282 \mathrm{~h} \mathrm{mo}^{-1}\right)$.

\section{Natural regeneration of Avicennia germinans}

The density of $A$. germinans plants showed differences between sites $(P<0.05)$. Density was highest at site 4 (reference), reaching 307 individuals per $0.01 \mathrm{ha}$, but decreased to 88 individuals at the end of the monitoring period. It was lowest at site 2, though it increased from 6.78 to 12.42 individuals per 0.01 ha during the monitoring period. Plant density at the restoration sites $(1,2$, and 3$)$ was lower than at the reference site (4); however, the mean value obtained for site 3 (50.95 individuals per $0.01 \mathrm{ha}$ ) was the closest to that obtained for site 4 (Fig. 2a).

There were no differences in the survival of $A$. germinans plants between sites. Survival at the restoration sites tended to increase over time, especially at site 1 , where it increased from $42.85 \%$ ( 0.7 years) to $99.28 \%$ (3.8 years). Survival values remained constant at sites $1-3$ over the 3.8 years, indicating the success of the restoration actions. Survival at the reference site (4) decreased from $100 \%$ to $29.79 \%$ (Fig. 2b).

Differences in plant height $(P<0.05)$ were observed between sites. At the end of the study, the highest mean value $(99.84 \mathrm{~cm})$ was recorded at site 4 (reference) and the lowest $(55 \mathrm{~cm})$ at site 1 . Within the restoration area, site 3 had the highest mean value $(66 \mathrm{~cm})$ (Fig. 2c).

There were no differences in RGRH between sites. During the first period (0.7 years), RGRH increased at all sites but was greater $\left(0.067 \mathrm{~mm} \mathrm{~d}^{-1}\right)$ at site 4 (reference). After 1.8 years, RGRH decreased at sites 2,3 , and 4 . The largest increase in RGRH occurred after 2.4 years, the highest value $\left(0.159 \mathrm{~mm} \mathrm{~d}^{-1}\right)$ corresponding to site 4 and the no presentaron cambios después de la restauración. El contenido de materia orgánica fue diferente entre sitios $(P<$ 0.0001). Los sitios de menor y mayor elevación determinaron los extremos de la variación ( $23 \pm 0.40 \%$ y $14 \pm 0.39 \%$, respectivamente) del contenido de materia orgánica (Tabla 1).

La elevación topográfica del sitio 4 fue de $+0.14 \pm 0.02$, la del sitio de mayor elevación fue de $+0.24 \pm 0.03 \mathrm{msnm}$ y la del sitio de menor elevación fue de $-0.04 \pm 0.01 \mathrm{msnm}$. La regeneración natural de $A$. germinans se presentó en un intervalo de altura del suelo de 0.087 a $0.14 \mathrm{~m}$ sobre el nivel medio del mar (Tabla 2). En los sitios de mayor y menor elevación no se establecieron plántulas.

\section{Características hidrológicas}

La salinidad varió entre sitios $(P<0.0001)$ y entre el tiempo de la restauración $(P<0.003)$. Después de las acciones de restauración, el sitio 4 (referencia) presentó el valor de salinidad más bajo $(64.50 \pm 1.80)$ y el sitio de mayor elevación presentó el valor más alto $(78.90 \pm 2.61)$ (Tabla 1).

El periodo y el nivel de inundación presentaron diferencias entre sitios $(P<0.05)$. También se observaron diferencias antes y después de las acciones de restauración $(P<0.05)$ (Tabla 2). La menor frecuencia de inundación se presentó en el sitio de menor elevación, donde se registraron 4 inundaciones por mes; en cambio, en los otros sitios se registraron entre 7 y 8 inundaciones por mes. El máximo nivel de inundación $(0.28 \mathrm{~m})$ se registró en el sitio de menor elevación, y el nivel mínimo $(0.08 \mathrm{~m})$ se registró en el sitio de mayor elevación. El nivel promedio de inundación en los sitios 1-3 fue de $0.14 \mathrm{~m}$ y en el sitio 4 (referencia) el nivel de inundación fue de $0.11 \mathrm{~m}$. El tiempo promedio de inundación disminuyó con el incremento de la elevación. El promedio más alto $\left(680 \mathrm{~h} \mathrm{mes}^{-1}\right)$ se observó en el sitio de elevación

Table 2. Variation of hydroperiod and porewater salinity at several mangrove sites (mean $\pm \mathrm{SE}$ ). Values for topographic elevation are meters above sea level (masl).

Tabla 2. Variación del hidroperiodo y la salinidad intersticial entre varios sitios de manglar (media \pm error estándar). Los valores para la elevación topográfica se muestran en metros sobre el nivel del mar (masl).

\begin{tabular}{|c|c|c|c|c|c|}
\hline Site & $\begin{array}{c}\text { Topographic } \\
\text { elevation } \\
\text { (masl) }\end{array}$ & $\begin{array}{l}\text { Water } \\
\text { level } \\
(\mathrm{m})\end{array}$ & $\begin{array}{l}\text { Flooding } \\
\text { duration } \\
\left(\mathrm{h} \mathrm{mo}^{-1}\right)\end{array}$ & $\begin{array}{l}\text { Frequency of } \\
\text { inundation } \\
\left(\text { no. tides } \mathrm{mo}^{-1}\right)\end{array}$ & $\begin{array}{c}\text { Porewater } \\
\text { salinity }\end{array}$ \\
\hline${ }^{1}$ Site 2 & $+0.09 \pm 0.02$ & $0.14 \pm 0.09$ & $524 \pm 4.44$ & $7 \pm 0.70$ & $76.79 \pm 3.16$ \\
\hline${ }^{1}$ Site 3 & $+0.08 \pm 0.01$ & $0.15 \pm 0.09$ & $529 \pm 4.40$ & $7 \pm 0.70$ & $69.01 \pm 2.21$ \\
\hline${ }^{1}$ Lower elevation & $-0.04 \pm 0.01$ & $0.28 \pm 0.10$ & $680 \pm 2.74$ & $4 \pm 0.58$ & $64.83 \pm 3.46$ \\
\hline${ }^{1}$ Higher elevation & $+0.24 \pm 0.03$ & $0.08 \pm 0.08$ & $282 \pm 4.33$ & $8 \pm 0.64$ & $80.88 \pm 3.57$ \\
\hline${ }^{2}$ Florida, USA & +0.46 & 0.18 & 424 & No data & 70 \\
\hline${ }^{3}$ Punta Guatales, Honduras & No data & 0.16 & 350 & 8 & 84.2 \\
\hline${ }^{4}$ Sinaloa, Mexico & 0.55 & 0.64 & No data & No data & 65 \\
\hline
\end{tabular}

Reference: ${ }^{1}$ this study; ${ }^{2}$ Lewis (2005), Chen and Twilley (1998); ${ }^{3}$ Castañeda-Moya et al. (2006); ${ }^{4}$ Flores-Verdugo et al. (2007). 
lowest $\left(0.11 \mathrm{~mm} \mathrm{~d}^{-1}\right)$ to site 1 . After 3.8 years, a decrease was observed at all sites, the values ranging from 0.078 to $0.087 \mathrm{~mm} \mathrm{~d}^{-1}$ (Fig. 2d).

\section{Environmental variables influencing regeneration}

We determined the relationship between the soil and hydrologic characteristics and the natural regeneration of A. germinans. Components RDA1 and RDA2 explained $91.2 \%$ of the total variance through new variables created from the original ones. RDA1 explained $64 \%$ of the variance, the most relevant variables being microtopography and hydroperiod (inundation frequency, water level, and flooding duration). RDA2 explained $27 \%$ of the variance, the most relevant variables being organic matter and porewater salinity (Table 3). Sites 1 and 4 correlated with the frequency of inundation and microtopography, explaining the response variables relative to plant density, height, and RGRH. Site 2 correlated with organic matter and porewater salinity. Site 3 correlated with water level and flooding duration. In this study, we determined that water level, flooding duration, inundation frequency, and topography were associated with the natural regeneration of A. germinans (Fig. 3).
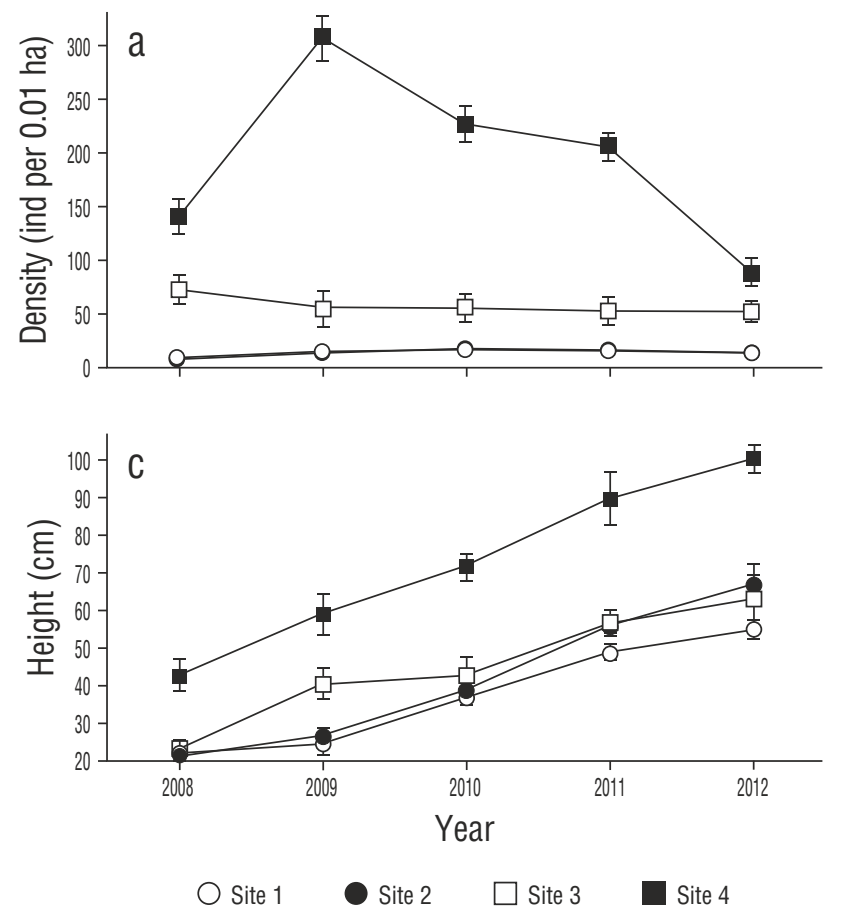

baja, seguido de los sitios en el área de restauración (sitios 1-3, 529-481 h mes ${ }^{-1}$ ), el sitio 4 (referencia, $439 \mathrm{~h} \mathrm{mes}^{-1}$ ) y el sitio de mayor elevación $\left(282 \mathrm{~h} \mathrm{mes}^{-1}\right)$.

\section{Regeneración natural de Avicennia germinans}

La densidad de individuos de A. germinans fue diferente entre sitios $(P<0.05)$. La mayor densidad se registró para el sitio 4 (referencia), donde la densidad fue de 307 individuos por cada 0.01 ha, pero disminuyó a 88 individuos al final del monitoreo. La menor densidad se registró para el sitio 2, que inicialmente era de 6.78 individuos por cada 0.01 ha y al finalizar el estudio aumentó a 12.42 individuos. Las densidades de A. germinas en los sitios de restauración (1-3) fueron menores que aquellas en el sitio de referencia (4); sin embargo, el valor de la densidad promedio en el sitio 3 (50.95 individuos por cada $0.01 \mathrm{ha}$ ) fue el más cercano a los valores del sitio 4 (Fig. 2a).

En cuanto a la supervivencia de los individuos de A. germinans, no se encontraron diferencias entre sitios. La supervivencia de los individuos en los sitios dentro del área de restauración tendió a aumentar con el transcurso del tiempo. Esta tendencia se observó mejor en el sitio 1, donde
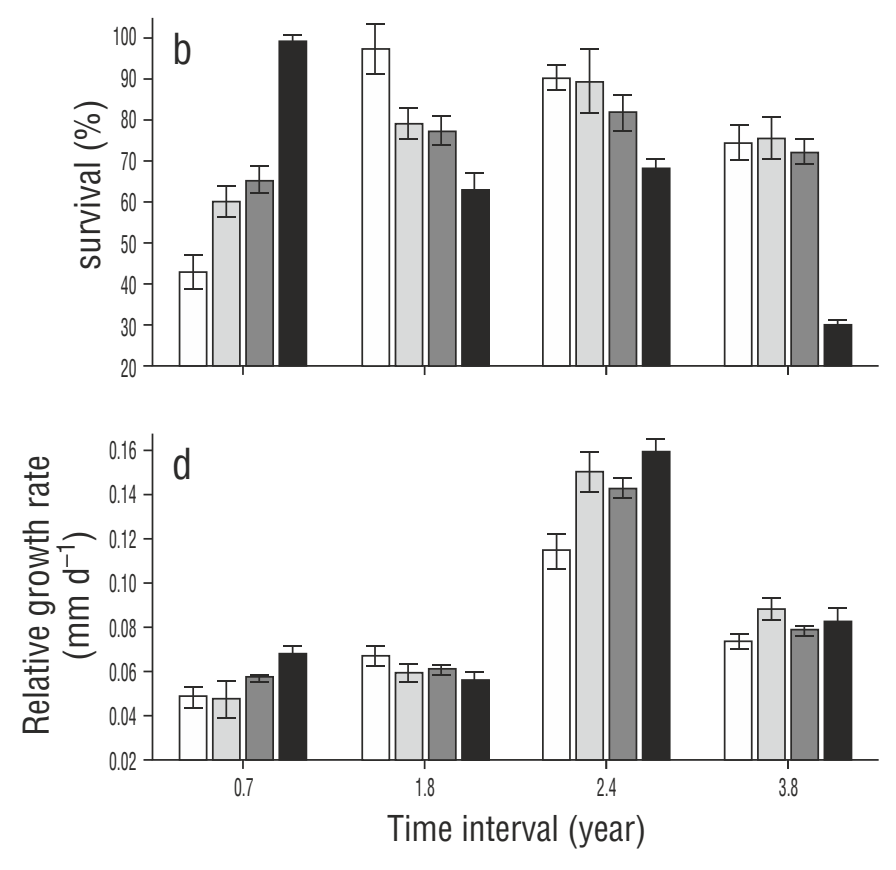

Figure 2. Variables used to assess the natural regeneration of Avicennia germinans: (a) density, (b) survival, (c) height, and (d) relative growth rate (mean \pm SE). Plant density and height are shown for the years 2008-2013. Survival and growth rate are shown for the following time intervals: $0.7,1.8,2.4$, and 3.8 years.

Figura 2. Variables utilizadas para evaluar la regeneración natural de Avicennia germinans: (a) densidad, (b) supervivencia, (c) altura y (d) tasa relativa de crecimiento (promedio \pm error estándar). La densidad y la altura de las plantas se muestra para los años 2008-2013. La supervivencia y tasa relativa de crecimiento se muestran con el cambio en el tiempo: $0.7,1.8,2.4$ y 3.8 años. 


\section{DISCUSSION}

Ecological restoration through hydrologic rehabilitation (desilting of tidal channels) and modification of the topography restored the environmental conditions at the sampling sites. The input of water to the restored sites led to changes in hydroperiod and soil conditions. Hydroperiod (water level, inundation frequency, and flooding duration) was important as it influenced the transport and settlement of propagules and the survival of mangrove seedlings.

The effect of the hydrological regime on mangrove regeneration in the restoration area indicated a differential trend. The establishment of $A$. germinans showed a patchy distribution, occurring only at the sites where the conditions were appropriate (Lewis 2005, Monroy-Torres et al. 2014, Spier et al. 2016). Not all the restoration area responded to the restoration actions at the same speed because the appropriate environmental conditions did not occur throughout the area. At the lower elevation site $(-0.040$ masl $)$, where water remained for a longer period of time $(680 \pm 2.74 \mathrm{~h})$ and the level was higher $(0.28 \pm 0.1 \mathrm{~m})$, propagules were unable to settle and develop because of the reduced oxygen conditions that affect physiological functions, such as obtaining water and nutrients through the roots (Krauss et al. 2008). Neither did natural regeneration occur at the higher elevation site ( 0.24 masl) because the propagules transported by the tide were unable to settle due to the high soil bulk density $\left(1.81 \mathrm{~g} \mathrm{~cm}^{-3}\right)$ (Naidoo 2006).

The decrease in porewater salinity at all the sites was a determining factor for mangrove growth (Fig. 4). Porewater salinity decreased as a result of the increased frequency of flooding that "cleaned" the soil (inflowing water had a salinity of 38 and outflowing water a salinity of 46) and improved water exchange (Krauss et al. 2006). At the restored sites, we observed short $(<70 \mathrm{~cm})$, flowering mangroves in juvenile stages, with lateral shrublike branch growth. In contrast, plant heights of $1 \mathrm{~m}$ were observed at site 4 (reference). The shorter plant height is associated with high salinity and bulk density (Bashan et al. 2013, Monroy-Torres et al. 2014, Flores-Verdugo et al. 2015). la supervivencia a los 0.7 años fue de $42.85 \%$ y luego aumentó a $99.28 \%$ a los 3.8 años. Todos los sitios mantuvieron valores constantes durante los 3.8 años, lo que puede indicar el éxito de las acciones de restauración alcanzado en el área de estudio. La supervivencia en el sitio de referencia (4) disminuyó del 100\% al 29.79\% (Fig. 2b).

Se observaron diferencias en la altura de los individuos de A. germinans $(P<0.05)$ entre sitios. Al final del estudio, la mayor altura promedio $(99.84 \mathrm{~cm})$ se registró para el sitio 4 (referencia) y la menor para el sitio $1(55 \mathrm{~cm})$. Dentro del área de restauración, la altura promedio $(66 \mathrm{~cm})$ fue mayor en el sitio 3 (Fig. 2c).

No se determinaron diferencias para la TRCA entre sitios. Durante el primer periodo ( 0.7 años), la TRCA incrementó en todos los sitios, y fue más alta $\left(0.067 \mathrm{~mm} \mathrm{~d}^{-1}\right)$ en el sitio 4 (referencia). Después de 1.8 años, la TRCA disminuyó en los sitios 2, 3 y 4 . El mayor incremento de la TRCA ocurrió a los 2.4 años, cuando la tasa más alta $\left(0.159 \mathrm{~mm} \mathrm{~d}^{-1}\right)$ fue registrada para el sitio 4 (referencia) y la más baja $\left(0.11 \mathrm{~mm} \mathrm{~d}^{-1}\right)$ para el sitio 1 (área de restauración). Después de 3.8 años, la TRCA disminuyó en todos los sitios, y los valores variaron entre 0.078 y $0.087 \mathrm{~mm} \mathrm{~d}^{-1}$ (Fig. 2d).

\section{Variables ambientales que determinan la regeneración natural}

Se determinó la relación de las características del suelo e hidrológicas con la regeneración natural de A. germinans. Los componentes RDA1 y RDA2 explicaron el $91.2 \%$ de la varianza total a través de nuevas variables generadas a partir de las originales. El RDA1 explicó el $64 \%$ de la varianza, y las variables más relevantes fueron la microtopografía y el hidroperiodo (frecuencia, nivel y periodo de inundación). El RDA2 explicó el $27 \%$ de la varianza, y las variables más relevantes fueron la materia orgánica y salinidad intersticial (Tabla 3). Los sitios 1 y 4 se correlacionaron con la frecuencia de inundaciones y la microtopografía, ya que explicaron las variables de respuesta con respecto a la densidad, altura y TRCA de A.germinans (Fig. 3). El sitio 2 se asoció a la materia orgánica y salinidad intersticial. El sitio 3 se

Table 3. Results of the redundancy analysis (RDA) according to components RDA1 and RDA2 of the environmental variables. The values in bold indicate the variables that contributed the most to natural regeneration.

Tabla 3. Resultados del análisis de redundancia (RDA) de acuerdo con los componentes RDA1 y RDA2 de las variables ambientales. Los valores en negritas indican las variables ambientales que más contribuyeron a la regeneración natural.

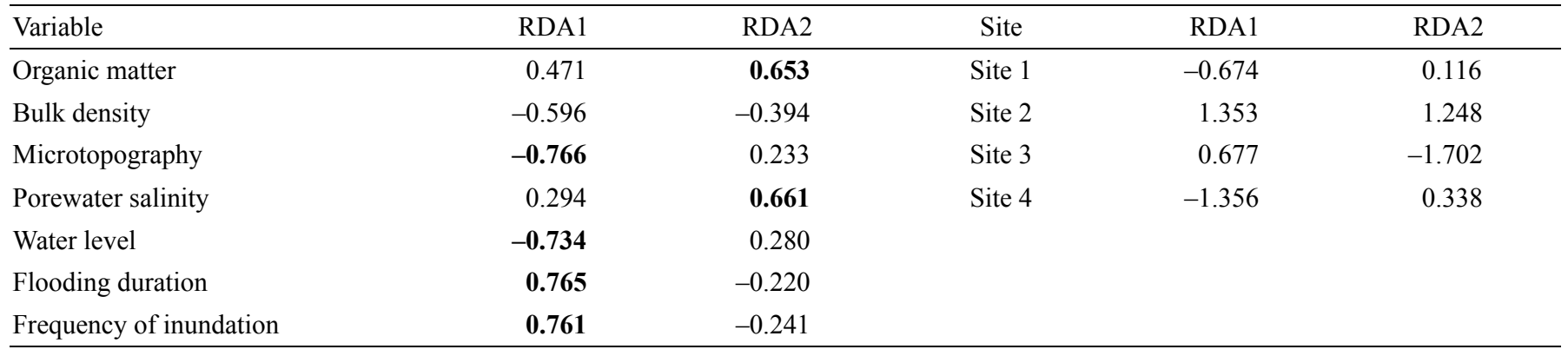




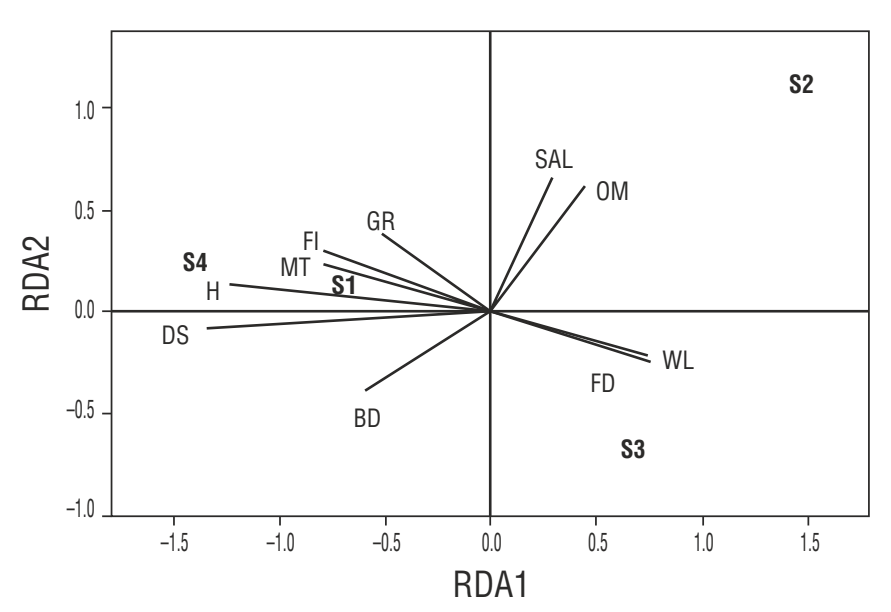

Figure 3. Redundancy analysis (RDA) plot according to components RDA1 and RDA2 of the environmental variables: bulk density (BD), organic matter (OM), microtopography (MT), porewater salinity (SAL), water level (WL), flooding duration (FD), and frequency of inundation (FI). The biological variables are seedling density (DS), height (H), and growth rate (GR). S1, S2, S3, and S4 refer to sites 1-4, respectively.

Figura 3. Gráfico del análisis de redundancia (RDA) de acuerdo con los componentes RDA1 y RDA2 de las variables ambientales: densidad aparente (BD), materia orgánica (OM), microtopografía (MT), salinidad intersticial (SAL), nivel de inundación (WL), periodo de inundación (FD), frecuencia de inundación (FI). Las variables biológicas son las siguientes: densidad de plántulas (DS), altura $(\mathrm{H})$ y tasa de crecimiento (GR). S1, S2, S3, y S4 indican los sitios de muestreo $1-4$, respectivamente.

Seedling density was higher at the reference site because of the presence of adult trees that produced propagules. Density at the restored sites, however, depended on the tide for propagule input, dispersion, and recruitment. This latter colonization process has been studied in other restoration areas where transport through tidal channels allowed seedling establishment (Lewis 2005).

Plant survival was greater at the restored sites (74\%) than at the reference sites (29\%). This difference can be attributed to 2 biological interactions of black mangrove seedlings. The plants at the reference sites showed a negative interaction associated with density-dependent mortality (Chu et al. 2010). Conversely, the plants at the restored sites showed a positive or facilitative interaction because seedlings benefit from their patchy distribution (Vogt et al. 2014), which improves nutrient availability (Corbin and Holl 2012), oxygenation, and soil elevation via root production (Milbrandt and Tinsley 2006).

For the natural regeneration of A. germinans, the optimum topographic elevation was 0.087 masl. In the case of hydroperiod, the optimum values were 8 floods per month, a flooding duration of $529 \mathrm{~h} \mathrm{~m}^{-1}$, and a water level of $0.14 \mathrm{~m}$. correlacionó con el nivel y periodo de inundación. Para este estudio, se determinó que el nivel, el periodo y la frecuencia de inundación, y la topografía se asociaron con la regeneración natural de A. germinans (Fig. 3).

\section{DISCUSIÓN}

La restauración ecológica mediante las rehabilitación hidrológica (desazolve de canales de marea) y la modificación de la topografía permitieron la recuperación de las condiciones ambientales de los sitios de muestreo. El ingreso del agua a los sitios de restauración dio como resultado cambios en el hidroperiodo y condiciones del suelo. El hidroperiodo (nivel, frecuencia y periodo de inundación) fue determinante, ya que influyó en el transporte y establecimiento de los propágulos y en la supervivencia de las plántulas de manglar.

El efecto del régimen hidrológico sobre la regeneración del manglar en el sitio de restauración demostró una tendencia diferencial. El establecimiento de A. germinans presentó una distribución en forma de parches, únicamente en los sitios donde las condiciones eran las adecuadas para su establecimiento (Lewis 2005, Monroy-Torres et al. 2014, Spier et al. 2016). Se comprobó que no toda el área de restauración respondió a la misma velocidad a las acciones de restauración, ya que el restablecimiento de las condiciones ambientales adecuadas no ocurrió en toda la zona. No se registró vegetación de manglar en el sitio de menor elevación $(-0.040 \mathrm{msnm})$ debido a que permaneció inundado durante mayor tiempo $(680 \pm 2.74 \mathrm{~h})$ y el nivel de inundación ahí fue el más alto $(0.28 \pm 0.1 \mathrm{~m})$; esto evitó que los propágulos pudieran establecerse y desarrollarse en condiciones reducidas de oxígeno, que afectan las funciones fisiológicas como la obtención del agua y nutrientes a través de las raíces (Krauss et al. 2008). En el sitio de mayor elevación $(0.24 \mathrm{msnm})$ tampoco hubo regeneración de manglar; esto se debió a que los propágulos que llegaron con la marea no se establecieron por la alta densidad aparente del suelo $\left(1.81 \mathrm{~g} \mathrm{~cm}^{-3}\right)$ (Naidoo 2006).

La reducción en la salinidad intersticial en todos los sitios fue un factor determinante para el crecimiento de A. germinans, incluso en el sitio de referencia (Fig. 4). Las frecuentes inundaciones provocaron un "lavado" del suelo (agua con salinidad de 38 al ingresar a la laguna y de 46 al salir), lo que mejoró el recambio del agua en el sitio y disminuyó la salinidad (Krauss et al. 2006). En los sitios restaurados, las plántulas presentaron características de mangle chaparro en etapa juvenil, ya que midieron no más de $70 \mathrm{~cm}$ de alto y sus ramas laterales crecieron en forma arbustiva; en cambio, en el sitio de referencia, la altura de las plántulas fue de $1 \mathrm{~m}$. El desarrollo de mangle chaparro se debió a las altas salinidades y densidades del suelo (Bashan et al. 2013, Monroy-Torres et al. 2014, Flores-Verdugo et al. 2015).

La densidad de plántulas fue mayor en el sitio de referencia debido a la presencia de árboles adultos que produjeron propágulos en este sitio. Sin embargo, la densidad en los 


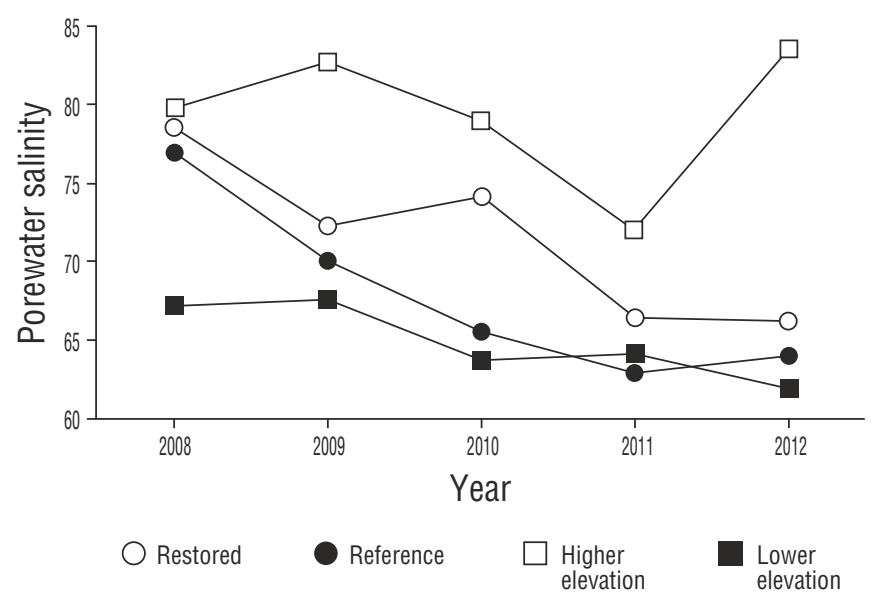

Figure 4. Interannual comparison of mean porewater salinity at the restored sites $(1,2$, and 3$)$, the reference site (4), and the higher and lower elevation sites.

Figura 4. Comparación interanual de la salinidad intersticial promedio en los sitios de restauración (1, 2 y 3$)$, el sitio de referencia (4) y los sitios de elevación tropográfica alta y baja.

Our findings show that the topographic gradients and hydroperiod characteristics in the restoration area were the most important environmental controllers of the natural regeneration of $A$. germinans. The restoration activities (modification of the microtopography and reestablishment of the hydroperiod) helped to reduce salinity, reestablish water flow and propagule dispersion, and contributed to the development of mangrove seedlings and juveniles. This study contributes to the knowledge of environmental regulators and the dynamics of mangrove forest development in restoration projects.

\section{ACKNOWLEDGMENTS}

This study was supported by the National Commission for the Knowledge and Use of Biodiversity (CONABIO, Mexico) and CINVESTAV at Mérida (project no. FB1338/ GH009/08). The first author acknowledges support from the National Council for Science and Technology (CONACYT, Mexico) through project 345.

English translation by Christine Harris.

\section{REFERENCES}

Adame MF, Zaldívar-Jiménez A, Teutli C, Caamal JP, Andueza MT, López-Adame H, Cano R, Hernández-Arana HA, Torres-Lara R, Herrera-Silveira JA. 2013. Drivers of mangrove litterfall within a karstic region affected by frequent hurricanes. Biotropica 45: 147-154. http://doi.org/10.1111/btp.12000

Bashan Y, Moreno M, Salazar BG, Alvarez L. 2013. Restoration and recovery of hurricane-damaged mangroves using the knickpoint retreat effect and tides as dredging tools. J. Environ. Manage. 116: 196-203. sitios de restauración dependió del flujo de marea, que ayudó al aporte, dispersión y reclutamiento de A. germinans. Este proceso de colonización también ha sido estudiado en áreas de restauración en donde la reconexión por canales de marea permitió el establecimiento de plántulas (Lewis 2005).

La supervivencia de A. germinans fue mayor en los sitios de restauración (74\%) que en los sitios de referencia (29\%). Esta diferencia se puede explicar por 2 interacciones biológicas de las plántulas de mangle negro. Los manglares en los sitios de referencia presentaron una interacción negativa debido a la mortalidad por densodependencia (Chu et al. 2010). Por el contrario, en los manglares de los sitios de restauración la interacción fue positiva o de facilitación, ya que las plántulas se beneficiaron al distribuirse en parches (Vogt et al. 2014) y mejoraron la disponibilidad de nutrientes (Corbin y Holl 2012), la oxigenación y la elevación del suelo a través de la producción de raíces (Milbrandt y Tinsley 2006).

Para la regeneración natural de $A$. germinans, la elevación topográfica óptima fue de $0.087 \mathrm{msnm}$. En el caso del hidroperiodo, los valores óptimos fueron 8 inundaciones al mes para la frecuencia de inundación, $529 \mathrm{~h}$ al mes para el periodo de inundación y $0.14 \mathrm{~m}$ para el nivel de inundación.

Los resultados de este estudio demostraron que los gradientes topográficos y las características del hidroperiodo en el área de restauración fueron los controladores ambientales más importantes para la regeneración natural del mangle negro. Las acciones de restauración (modificación de la microtopografía y restablecimiento del hidroperiodo) contribuyeron a la disminución de la salinidad, el restablecimiento del flujo de agua, la dispersión de propágulos y el desarrollo de plántulas y juveniles de mangle. Este trabajo contribuye al conocimiento de la importancia de los reguladores ambientales y la dinámica del desarrollo de la vegetación de manglar en los programas de restauración.

\section{AgRadecimientos}

Este estudio fue financiado por la Comisión Nacional para el Conocimiento y Uso de la Biodiversidad (CONABIO, México) y el CINVESTAV, unidad Mérida, a través del proyecto no. FB1338/GH009/08. La primera autora agradece al Consejo Nacional de Ciencia y Tecnología (CONACYT, México) la Cátedra otorgada a través del proyecto 345.

Bosire JO, Dahdouh-Guebas F, Walton M, Crona, BI, Lewis RR, Field C, Kairo JG, Koedam N. 2008. Functionality of restored mangroves: A review. Aquat. Bot. 89: 251-259.

Cardona-Olarte P, Twilley RR, Krauss KW, Rivera-Monroy V. 2006. Responses of neotropical mangrove seedlings grown in monoculture and mixed culture under treatments of hydroperiod and salinity. Hydrobiologia 569(1): 325-341.

Castañeda-Moya E, Rivera-Monroy VH, Twilley RR. 2006. Mangrove zonation in the dry life zone of the Gulf of Fonseca, Honduras. Estuaries Coasts 29(5): 751-764. 
Chen R, Twilley RR. 1998. A gap dynamic model of mangrove forest development along gradients of soil salinity and nutrient resources. J. Ecol. 86(1): 37-51.

Chen R, Twilley RR. 1999. A simulation model of organic matter and nutrient accumulation in mangrove wetland soils. Biogeochemistry 44(1): 93-118.

Chu CJ, Weiner J, Maestre FT, Wang YS, Morris C, Xiao S, Wang G. 2010. Effects of positive interactions, size symmetry of competition and abiotic stress on self-thinning in simulated plant populations. Ann. Bot. London 106(4): 647-652.

Corbin JD, Holl KD. 2012. Applied nucleation as a forest restoration strategy. Forest Ecol. Manage. 265: 37-64.

Dahdouh-Guebas F, Kairo JG, De Bond R, Koedam N. 2007. Pheumatophore height and density in relation to microtopography in the grey mangrove Avicennia marina. Belg. J. Bot. 140(2): 213-221.

Feller IC, Lovelock CE, Berger U, McKee KL, Joye SB, Ball MC. 2010. Biocomplexity in mangrove ecosystems. Annu. Rev. Mar. Sci. 2: 395-417.

Flores-Verdugo FJ, Agraz-Hernández CM, Benítez-Pardo D. 2006. Ecosistemas acuáticos costeros: Importancia, retos y prioridades para su conservación. In: Sánchez O, Herzig M, Peters RE, Márquez-Huitzil R, Zambrano L (eds.), Perspectivas sobre conservación de ecosistemas acuáticos en México. Instituto Nacional de Ecología, México, 294 pp.

Flores-Verdugo FJ, Moreno-Casasola $\mathrm{P}$, Agraz-Hernández $\mathrm{M}$, López-Rosas H, Benítez-Pardo D, Travieso-Bello AC. 2007. La topografía y el hidroperíodo: Dos factores que condicionan la restauración de los humedales costeros. Bol. Soc. Bot. México (Sup.): 33-47.

Flores-Verdugo F, Zebadua-Penagos F, Flores-de-Santiago F. 2015. Assessing the influence of artificially constructed channels in the growth of afforested black mangrove (Avicennia germinans) within an arid coastal region. J. Environ. Manage. 160: 113-120.

García de Fuentes A, Xool Koh M, Euán-Ávila JI, Munguía-Gil A, Cervera Montejano MD. 2011. La costa de Yucatán en la perspectiva del desarrollo turístico. Comisión Nacional para el Conocimiento y Uso de la Biodiversidad, Colección Corredor Biológico Mesoamericano, México, Serie Conocimientos 9, México, DF, 82 pp.

He B, Lai T, Fan H, Wang W, Zheng H. 2007. Comparison of flooding-tolerance in four mangrove species in a diurnal tidal zone in the Beibu Gulf. Estuar. Coast. Shelf Sci. 74: 254-262.

Kitaya Y, Sumiyoshi M, Kawabata Y, Monji N. 2002. Effect of submergence and shading of hypocotyls on leaf conductance in young seedlings of the mangrove Rhizophora stylosa. Trees 16(2-3): 147-149.

Komiyama A, Santiean T, Higo M, Patanaponpaiboon P, Kongsangchai J, Ogino K. 1996. Microtopography, soil hardness and survival of mangrove (Rhizophora apiculata BL.) seedlings planted in an abandoned tin-mining area. Forest Ecol. Manage. 81(1-3): 243-248.

Krauss KW, Doyle TW, Twilley RR, Rivera-Monroy VH, Sullivan JK. 2006. Evaluating the relative contributions of hydroperiod and soil fertility on growth of south Florida mangroves. Hydrobiologia 569: 311-324.

Krauss KW, Lovelock CE, McKee KL, Lopez-Hoffman L, Ewe SML, Sousa WP. 2008. Environmental drivers in mangrove establishment and early development: A review. Aquat. Bot. 89(2): 105-127.

Kuenzer C, Bluemel A, Gebhardt S, Quoc TV, Dech S. 2011. Remote sensing of mangrove ecosystems: A review. Remote Sens. 3(12): 878-928.
Legendre P, Legendre L. 1998. Numerical Ecology. 2nd ed. Elsevier Science, Amsterdam.

Lewis RR. 2005. Ecological engineering for successful management and restoration of mangrove forests. Ecol. Eng. 24(4): 403-418.

McKee KL. 1995. Seedling recruitment patterns in a Belizean mangrove forest: Effects of establishment ability and physicochemical factors. Oecologia 101(4): 448-460.

Milbrandt EC, Tinsley MN. 2006. The role of saltwort (Batis maritima L.) in regeneration of degraded mangrove forests. Hydrobiologia 568: 369-377.

Monroy-Torres M, Flores-Verdugo F, Flores-de-Santiago F. 2014. Growth of three subtropical mangrove species in response to varying hydroperiod in an experimental tank $=$ Crecimiento de tres especies de mangle subtropical en respuesta a la variabilidad en el hidroperiodo en un tanque experimental. Cienc. Mar. 40(4): 263-275. http://dx.doi.org/10.7773/cm.v40i4.2455

Moroyoqui-Rojo L, Flores-Verdugo F, Escobedo-Urias DC, Floresde-Santiago F, González-Farías F. 2015. Potential use of two subtropical mangrove species (Laguncularia racemosa and Rhizophora mangle) for nutrient removal in closed recirculating systems = Uso potencial de dos especies de mangle subtropical (Laguncularia racemosa y Rhizophora mangle) para la remoción de nutrientes en sistemas de recirculación cerrados. Cienc. Mar. 41(4): 255-268. http://dx.doi.org/10.7773/cm.v41i4.2521

Naidoo G. 2006. Factors contributing to dwarfing in the mangrove Avicennia marina. Ann. Bot. London 97(6): 1095-1101.

Orellana R, Espadas IG. 2003. Presente, pasado y futuro de los climas de la Península de Yucatán. In: Colunga-García A, Larqué-Saavedra MP (eds.), Naturaleza y Sociedad en el Área Maya. Academia Mexicana de Ciencias-CICY, pp. 37-52.

R Core Team. 2012. R: A Language and Environment for Statistical Computing. R Foundation for Statistical Computing, Vienna, Austria.

Reef R, Feller IC, Lovelock CE. 2010. Nutrition of mangroves. Tree Physiol. 30: 1148-1160.

Ricklefs RE, Miller GL, 2000. Ecology. 4th ed. WH Freeman \& Co, New York, 896 pp.

Rodríguez-Ramírez A, Nivia-Ruíz J, Garzón-Ferreira J. 2004. Structural and functional characteristics of Avicennia germinans mangrove formation of Chengue Bay (Colombian Caribbean). Bol. Invest. Mar. Cost. 33(1): 223-244.

[SER] Society for Ecological Restoration International, Grupo de trabajo sobre ciencia y políticas. 2004. Principios de SER International sobre la restauración ecológica. www.ser.org and Society for Ecological Restoration International, Tucson, AZ, $16 \mathrm{pp}$.

Sousa W, Quek SP, Mitchell BJ. 2003. Regeneration of Rhizophora mangle in a Caribbean mangrove forest: Interacting effects of canopy disturbance and a stem-boring beetle. Oecologia 137(3): 436-445.

Spier D, Gerum H, Noernberg MA, Lana PC. 2016. Flood regime as a driver of the distribution of mangrove and salt marsh species in a subtropical estuary. J. Mar. Syst. 161: 11-25.

Tabachnick BG, Fidell LS. 1996. Using Multivariate Statistics. 3rd ed. HarperCollins, New York, 880 pp.

Teutli-Hernández C. 2008. Regeneración de zonas de manglar bajo diferentes regímenes hidrológicos en sistemas cársticoscarbonatados. MSc thesis, Centro de Investigación y de Estudios Avanzados del Instituto Politécnico Nacional, Unidad Mérida, Yucatán, México. 
Twilley RR, Rivera-Monroy VH. 2005. Developing performance measures of mangrove wetlands using simulation models of hydrology, nutrient biogeochemistry, and community dynamics. J. Coast. Res. SI40: 79-93.

Vaslet A, Bouchon-Navaro Y, Harmelin-Vivien M, Lepoint G, Louis M, Bouchon C. 2015. Foraging habits of reef fishes associated with mangroves and seagrass beds in a Caribbean lagoon: A stable isotope approach $=$ Hábitos de alimentación de peces arrecifales asociados con manglares y pastos marinos en una laguna del Caribe: Un enfoque de isótopos estables. Cienc. Mar. 41(3): 217-232.

http://dx.doi.org/10.7773/cm.v41i3.2494
Vogt J, Lin Y, Pranchai A, Frohberg P, Mehlig U, Berger U. 2014. The importance of conspecific facilitation during recruitment and regeneration: A case study in degraded mangroves. Basic Appl. Ecol. 15(8): 651-660.

Whigham DF, Verhoeven JTA, Samarkin V, Megonigal PJ. 2009. Responses of Avicennia germinans (black mangrove) and the soil microbial community to nitrogen addition in a hypersaline wetland. Estuaries Coasts 32(5): 926-936.

Zaldívar-Jiménez MA, Herrera-Silveira JA, Teutli-Hernández C, Comín FA, Andrade JL, Coronado-Molina C, Pérez-Ceballos R. 2010. Conceptual framework for mangrove restoration in the Yucatán Peninsula. Ecol. Restor. 28(3): 333-342.

Received September 2016, accepted December 2016. 